TAMKANG JOURNAL OF MATHEMATICS

Volume 33, Number 3, Autumn 2002

\title{
ON COMPATIBLE MAPPINGS SATISFYING AN IMPLICIT RELATION IN COMMON FIXED POINT CONSIDERATION
}

\author{
SUSHIL SHARMA AND BHAVANA DESHPANDE
}

\begin{abstract}
In this paper, we prove some common fixed point theorems for compatible mappings satisfying an implicit relation.
\end{abstract}

\section{Introduction}

Wang, Li, Gao and Iseki [14] proved some fixed point theorems on expansion mappings which correspond to some contractive mappings. In a paper Rhoades [9] generalized the results for pairs of mappings. Some theorems on unique fixed point for expansion mappings are proved by Popa [6]. Popa [7] further extended results [6], [9] for compatible mappings.

In 1999, Popa [8] proved some fixed point theorems for compatible mappings satisfying an implicit relation.

Let $S$ and $T$ be two self mappings of a metric space $(X, d)$. Sessa [10] defines $S$ and $T$ to be weakly commuting if $d(S T x, T S x) \leq d(T x, S x)$ for all $x$ in $X$. Jungck [1] defines $S$ and $T$ to be compatible if $\lim _{n \rightarrow \infty} d\left(S T x_{n}, T S x_{n}\right)=0$ whenever $\left\{x_{n}\right\}$ is a sequence in $X$ such that $\lim _{n \rightarrow \infty} S x_{n}=\lim _{n \rightarrow \infty} T x_{n}=x$ for some $x$ in $X$. Clearly, commuting mappings are weakly commuting and weakly commuting mappings are compatible, but implications are not reversible [11, Ex 1] and [1, Ex 2.2].

Many authors have proved common fixed point theorems for compatible mappings for this we refer to Jungck [1], [2] and [3], Sessa, Rhoades and Khan [12], Kang, Cho and Jungck [4], Kang and Ray [5] and Sharma and Patidar [13].

In this paper, we prove common fixed point theorems for compatible mappings in Banach spaces, satisfying an implicit relation. We improve and generalize the results of Popa [6], [7] and [8].

Lemma 1.([1]) Let $S$ and $T$ be compatible self mappings on a metric space $(X, d)$. If $S(t)=T(t)$ for some $t \in X$ then $S T(t)=T S(t)$.

Received April 27, 2001; revised November 14, 2001.

2000 Mathematics Subject Classification. 47H10, 54H25

Key words and phrases. Compatible mappings, implicit relation, common fixed point. 


\section{Implicit Relations}

Let $\Phi$ be the set of all real continuous functions $\phi\left(t_{1}, t_{2}, \ldots, t_{6}\right): R_{+}^{6} \rightarrow R$ satifying the following conditions:

$\phi_{1}: \phi$ is non-increasing in variable $t_{6}$,

$\phi_{2}$ : there exists $h \in(0,1)$ such that for every $u, v \geq 0$ with

$$
\left(\phi_{a}\right): \phi(u, v, v, u,(1 / 2)(u+v), 0) \leq 0
$$

or

$$
\left(\phi_{b}\right): \phi(u, v, u, v,(1 / 2)(u+v), u+v) \leq 0
$$

we have $u \leq h v$.

$\phi_{3}: \phi(u, u, 0,0,0, u)>0$ for all $u>0$.

Example 1. $\phi\left(t_{1}, \ldots, t_{6}\right)=t_{1}-k \max \left\{t_{2}, t_{3}, t_{4}, t_{5},(1 / 2) t_{6}\right\}$, where $k \in(0,1)$.

$\phi_{1}$ : Obviously.

$\phi_{2}$ : Let $u>0, \phi(u, v, v, u,(1 / 2)(u+v), 0)=u-k \max \{v, v, u,(1 / 2)(u+v), 0\} \leq 0$. If

$u \geq v$ then $u \leq k u<u$, a contradiction. Thus $u<v$ and $u \leq k v=h v$, where $h=k \in(0,1)$.

Similarly, if $u>0$ then $\phi(u, v, u, v,(1 / 2)(u+v), u+v) \leq 0$ imply $u \leq h v$. If $u=0$, then $u \leq h v$.

$\phi_{3}: \phi(u, u, 0,0,0, u)=(1-k) u>0$, for all $u>0$.

Example 2. $\phi\left(t_{1}, t_{2}, \ldots, t_{6}\right)=t_{1}^{2}-a\left\{t_{2}^{2}-t_{6}\left((1 / 2)\left(t_{3}+t_{4}\right)-t_{5}\right)\right\}$, where $a \in(0,1)$. $\phi_{1}$ : Obviously.

$\phi_{2}$ : Let $u>0, \phi(u, v, v, u,(1 / 2)(u+v), 0)=u^{2}-a v^{2} \leq 0$, which implies $u \leq a^{1 / 2} v=h v$, where $h=a^{1 / 2}<1$.

Similarly, if $u>0$ then $\phi(u, v, u, v,(1 / 2)(u+v), u+v) \leq 0$ imply $u \leq h v$. If $u=0$, then $u \leq h v$.

$\phi_{3}: \phi(u, u, 0,0,0, u)=u^{2}(1-a)>0$, for all $u>0$.

Example 3. $\phi\left(t_{1}, \ldots, t_{6}\right)=t_{1}^{2}-c_{1} \max \left\{t_{2}^{2}, t_{3}^{2}, t_{4}^{2}\right\}-c_{2} \max \left\{t_{3} t_{5},(1 / 2) t_{4} t_{6}\right\}$, where $c_{1}>0, c_{2} \geq 0, c_{1}+c_{2}<1$.

$\phi_{1}$ : Obviously.

$\phi_{2}$ : Let $u>0, \phi(u, v, v, u,(1 / 2)(u+v), 0)=u^{2}-c_{1} \max \left\{v^{2}, v^{2}, u^{2}\right\}-c_{2} \max \{v(1 / 2)(u+$ $v), 0\} \leq 0$. If $u \geq v$ then $u^{2}\left(1-c_{1}-c_{2}\right) \leq 0$, which implies $c_{1}+c_{2} \geq 1$, a contradiction. Thus $u<v$ and $u \leq\left(c_{1}+c_{2}\right)^{1 / 2} v=h v$, where $h=\left(c_{1}+c_{2}\right)^{1 / 2}<1$. Similarly, if $u>0$ then $\phi(u, v, u, v,(1 / 2)(u+v), u+v) \leq 0$ imply $u \leq h v$. If $u=0$, then $u \leq h v$.

$\phi_{3}: \phi(u, u, 0,0,0, u)=u^{2}\left(1-c_{1}\right)>0$, for all $u>0$.

\section{Main Results}

Theorem 3.1. Let $(X,\|\cdot\|)$ be a Banach space and $A, B, S, T: X \rightarrow X$ be four mappings satisfying the conditions:

$$
\begin{array}{r}
\phi(\|A x-B y\|,\|S x-T y\|,\|S x-A x\|,\|T y-B y\|, \\
\quad(1 / 2)(\|S x-A x\|+\|T y-B y\|),\|T y-A x\|) \leq 0
\end{array}
$$


for all $x, y$ in $X$, where $\phi \in \Phi$,

$$
\begin{aligned}
& A(X) \subset T(X) \text { and } B(X) \subset S(X), \\
& \text { one of } A, B, S, T \text { is continuous, } \\
& \{A, S\} \text { and }\{B, T\} \text { are compatible pairs. }
\end{aligned}
$$

Then $A, B, S$ and $T$ have a unique common fixed point.

Proof. By (3.2), since $A(X) \subset T(X)$, for an arbitrary point $x_{0} \in X$ there exists a point $x_{1} \in X$ such that $A x_{0}=T x_{1}$. Since $B(X) \subset S(X)$, for this point $x_{1} \in X$, we can choose a point $x_{2} \in X$ such that $B x_{1}=S x_{2}$ and so on. Inductively we can define a sequence $\left\{y_{n}\right\}$ in $X$ such that

$$
y_{2 n}=T x_{2 n+1}=A x_{2 n} \text { and } y_{2 n+1}=S x_{2 n+2}=B x_{2 n+1}
$$

for every $n=0,1,2, \ldots$.

By (3.1), we have

$$
\begin{aligned}
\phi & \left(\left\|A x_{2 n}-B x_{2 n+1}\right\|,\left\|S x_{2 n}-T x_{2 n+1}\right\|,\left\|S x_{2 n}-A x_{2 n}\right\|,\left\|T x_{2 n+1}-B x_{2 n+1}\right\|,\right. \\
& \left.(1 / 2)\left(\left\|S x_{2 n}-A x_{2 n}\right\|+\left\|T x_{2 n+1}-B x_{2 n+1}\right\|\right),\left\|T x_{2 n+1}-A x_{2 n}\right\|\right) \leq 0 \\
\phi & \left(\left\|A x_{2 n}-B x_{2 n+1}\right\|,\left\|B x_{2 n-1}-A x_{2 n}\right\|,\left\|B x_{2 n-1}-A x_{2 n}\right\|,\right. \\
& \left.\left\|A x_{2 n}-B x_{2 n+1}\right\|,(1 / 2)\left(\left\|B x_{2 n-1}-A x_{2 n}\right\|+\left\|A x_{2 n}-B x_{2 n+1}\right\|\right), 0\right) \leq 0 .
\end{aligned}
$$

By $\left(\phi_{a}\right)$, we have

$$
\left\|A x_{2 n}-B x_{2 n+1}\right\| \leq h\left\|B x_{2 n-1}-A x_{2 n}\right\| .
$$

Similarly by $\left(\phi_{b}\right)$ and $\phi_{1}$, we have

$$
\left\|A x_{2 n}-B x_{2 n-1}\right\| \leq h\left\|A x_{2 n-2}-B x_{2 n-1}\right\| .
$$

and so

$$
\left\|A x_{2 n}-B x_{2 n-1}\right\| \leq h^{2 n}\left\|A x_{0}-B x_{1}\right\| \text { for } n=0,1,2, \ldots
$$

By a routine calculation it follows that $\left\{y_{n}\right\}$ is a Cauchy sequence in $X$ and hence it converges to a point $z$ in $X$. Consequently, subsequences $\left\{A x_{2 n}\right\},\left\{B x_{2 n+1}\right\},\left\{S x_{2 n}\right\}$ and $\left\{T x_{2 n+1}\right\}$ of $\left\{y_{n}\right\}$ also converges to the point $z$.

Let us now suppose that $S$ is continuous, so the sequence $\left\{S A x_{2 n}\right\}$ converges to $\{S z\}$. We have

$$
\left\|A S x_{2 n}-S z\right\| \leq\left\|A S x_{2 n}-S A x_{2 n}\right\|+\left\|S A x_{2 n}-S z\right\| .
$$

Since $S$ is continuous and $A$ and $S$ are compatible, letting $n$ tends to infinity, we state that the sequence $\left\{A S x_{2 n}\right\}$ also converges to $S z$. Using (3.1), we have

$$
\begin{gathered}
\phi\left(\left\|A S x_{2 n}-B x_{2 n+1}\right\|,\left\|S S x_{2 n}-T x_{2 n+1}\right\|,\left\|S S x_{2 n}-A S x_{2 n}\right\|,\left\|T x_{2 n+1}-B x_{2 n+1}\right\|,\right. \\
\left.\quad(1 / 2)\left(\left\|S S x_{2 n}-A S x_{2 n}\right\|+\left\|T x_{2 n+1}-B x_{2 n+1}\right\|\right),\left\|T x_{2 n+1}-A S x_{2 n}\right\|\right) \leq 0 .
\end{gathered}
$$


Letting $n$ tends to infinity we have by the continuity of $\phi$,

$$
\phi(\|S z-z\|,\|S z-z\|, 0,0,0,\|z-S z\|) \leq 0
$$

which is a contradiction to $\phi_{3}$ if $\|z-S z\| \neq 0$. Thus $S z=z$. Further by (3.1), we have

$$
\begin{gathered}
\phi\left(\left\|A z-B x_{2 n+1}\right\|,\left\|S z-T x_{2 n+1}\right\|,\|S z-A z\|,\left\|T x_{2 n+1}-B x_{2 n+1}\right\|,\right. \\
\left.\quad(1 / 2)\left(\|S z-A z\|+\left\|T x_{2 n+1}-B x_{2 n+1}\right\|\right),\left\|T x_{2 n+1}-A z\right\|\right) \leq 0 .
\end{gathered}
$$

Letting $n$ tends to infinity we have by the continuity of $\phi$,

$$
\phi(\|A z-z\|, 0,\|z-A z\|, 0,(1 / 2)\|z-A z\|,\|z-A z\|) \leq 0,
$$

which implies by $\left(\phi_{b}\right)$, that $A z=z$. This means that $z$ is in the range of $A$ and since $A(X) \subset T(X)$, there exists a point $u$ in $X$ such that $T u=z$.

Using (3.1), we have

$$
\begin{aligned}
\phi & (\|A z-B u\|,\|S z-T u\|,\|S z-A z\|,\|T u-B u\|, \\
& (1 / 2)(\|S z-A z\|+\|T u-B u\|),\|T u-A z\|) \leq 0 \\
= & \phi(\|z-B u\|, 0,0,\|z-B u\|,(1 / 2)\|z-B u\|, 0) \leq 0,
\end{aligned}
$$

which implies by $\left(\phi_{a}\right)$, that $z=B u$.

Since $T u=B u=z$, by Lemma 1 , it follows that $B T u=T B u$ and so $B z=B T u=$ $T B u=T z$.

Thus from (3.1), we have

$$
\begin{aligned}
\phi & (\|A z-B z\|,\|S z-T z\|,\|S z-A z\|,\|T z-B z\|, \\
& (1 / 2)(\|S z-A z\|+\|T z-B z\|),\|T z-A z\|) \leq 0 \\
= & \phi(\|z-T z\|,\|z-T z\|, 0,0,0,\|T z-z\|) \leq 0,
\end{aligned}
$$

which is contradiction to $\phi_{3}$ if $\|T z-z\| \neq 0$. Thus $T z=z=B z$.

We have therefore, proved that $z$ is a common fixed point of $A, B, S$ and $T$. The same result holds if $T$ is continuous instead of $S$. Now suppose that $A$ is continuous. Then the sequence $\left\{A S x_{2 n}\right\}$ converges to $A z$ we have

$$
\left\|S A x_{2 n}-A z\right\| \leq\left\|S A x_{2 n}-A S x_{2 n}\right\|+\left\|A S x_{2 n}-A z\right\| .
$$

Since $A$ is continuous and $A$ and $S$ are compatible, letting $n$ tends to infinity we obtain that $\left\{S A x_{2 n}\right\}$ converges to $A z$. Using (3.1), we have

$$
\begin{aligned}
\phi & \left(\left\|A A x_{2 n}-B x_{2 n+1}\right\|,\left\|S A x_{2 n}-T x_{2 n+1}\right\|,\left\|S A x_{2 n}-A A x_{2 n}\right\|,\left\|T x_{2 n+1}-B x_{2 n+1}\right\|,\right. \\
& \left.(1 / 2)\left(\left\|S A x_{2 n}-A A x_{2 n}\right\|+\left\|T x_{2 n+1}-B x_{2 n+1}\right\|\right), \| T x_{2 n+1}-A A x_{2 n}\right) \leq 0 .
\end{aligned}
$$

Letting $n$ tends to infinity, we have by continuity of $\phi$

$$
\phi(\|A z-z\|,\|A z-z\|, 0,0,0,\|z-A z\|) \leq 0,
$$


a contradiction to $\phi_{3}$ if $z \neq A z$. Thus $z=A z$.

This means that $z$ is in the range of $A$ and since $A(X) \subset T(X)$, there exists a point $v$ in $X$ such that $T v=z$. Thus by (3.1), we have

$$
\begin{gathered}
\phi\left(\left\|A A x_{2 n}-B v\right\|,\left\|S A x_{2 n}-T v\right\|,\left\|S A x_{2 n}-A A x_{2 n}\right\|,\|T v-B v\|,\right. \\
\left.(1 / 2)\left(\left\|S A x_{2 n}-A A x_{2 n}\right\|+\|T v-B v\|\right),\left\|T v-A A x_{2 n}\right\|\right) \leq 0 .
\end{gathered}
$$

Letting $n$ tends to infinity we get

$$
\phi(\|z-B v\|, 0,0,\|z-B v\|,(1 / 2)\|z-B v\|, 0) \leq 0
$$

and by $\left(\phi_{a}\right)$ it follows that $z=B v$. Since $T v=B v=z$, by Lemma 1 , it follows that $B z=B T v=T B v=T z$. Thus from (3.1) we have

$$
\begin{gathered}
\phi\left(\left\|A x_{2 n}-B z\right\|,\left\|S x_{2 n}-T z\right\|,\left\|S x_{2 n}-A x_{2 n}\right\|,\|T z-B z\|,\right. \\
\left.(1 / 2)\left(S x_{2 n}-A x_{2 n}\|+\| T z-B z \|\right), \| T z-A x_{2 n}\right) \leq 0 .
\end{gathered}
$$

Letting $n$ tends to infinity, we obtain

$$
\phi(\|z-B z\|,\|z-B z\|, 0,0,0,\|B z-z\|) \leq 0,
$$

which is contradiction to $\phi_{3}$, it follows that $z=B z=T z$. This means that $z$ is in the range of $B$ and since $B(X) \subset S(X)$ there exists $w \in X$ such that $S w=z$. Thus from (3.1) we have

$$
\begin{aligned}
& \phi(\|A w-B z\|,\|S w-T z\|,\|S w-A w\|,\|T z-B z\|, \\
& \quad(1 / 2)(\|S w-A w\|+\|T z-B z\|),\|T z-A w\|) \leq 0 . \\
& \phi(\|A w-z\|, 0,\|z-A w\|, 0,(1 / 2)\|z-A w\|,\|z-A w\|) \leq 0,
\end{aligned}
$$

and by $\left(\phi_{b}\right)$, we have $z=A w=S w$. Since $A w=S w=z$, by Lemma 1, it follows that $S A w=A S w$ and so $S z=S A w=A S w=A z=z$. We have therefore proved that $z$ is a common fixed point of $A, B, S$ and $T$.

The same result holds, if we assume that $B$ is continuous instead of $A$. By (3.1) and $\phi_{3}$ it follows that $z$ is unique.

Theorem 3.1 and Examples 1 to 3 imply the following:

Corollary 3.2. Let $(X,\|\cdot\|)$ be a Banach space and $A, B, S$ and $T: X \rightarrow X$ be four mappings satisfying the conditions (3.2) to (3.4) and the following:

$$
\begin{aligned}
\|A x-B y\| \leq & k \max \{\|S x-T y\|,\|S x-A x\|,\|T y-B y\|, \\
& (1 / 2)(\|S x-A x\|+\|T y-B y\|),(1 / 2)\|T y-A x\|\}
\end{aligned}
$$

for all $x, y$ in $X$, where $k \in(0,1)$,

or

$$
\|A x-B y\|^{2} \leq a\|S x-T y\|^{2}
$$


for all $x, y$ in $X$, where $a \in(0,1)$,

or

$$
\begin{aligned}
\|A x-B y\|^{2} \leq & c_{1} \max \left\{\|S x-T y\|^{2},\|S x-A x\|^{2},\|T y-B y\|^{2}\right\} \\
& +c_{2} \max \{\|S x-A x\|(1 / 2)(\|S x-A x\|+\|T y-B y\|), \\
& (1 / 2)\|T y-B y\|\|T y-A x\|\}
\end{aligned}
$$

for all $x, y$ in $X$, where $c_{1}>0, c_{2} \geq 0, c_{1}+c_{2}<1$.

Then $A, B, S$ and $T$ have a unique common fixed point.

We now give an example to illustrate the above results:

Example 4. Consider $X=[1,15]$ with the usual norm.

Define $A, B, S$ and $T$ by

$$
\begin{gathered}
A x=1 \text { if } x \in[1,15] \\
B x= \begin{cases}1 & \text { if } x=1, x>3 \\
2 & \text { if } 1<x \leq 3\end{cases} \\
S x= \begin{cases}1 & \text { if } x=1, x>3, \quad x \neq 12 \\
2 & \text { if } x=12 \\
15 & \text { if } 1<x \leq 3\end{cases} \\
T x= \begin{cases}1 & \text { if } x=1, x>3 \\
5 & \text { if } 1<x \leq 3\end{cases}
\end{gathered}
$$

Then $A, B, S$ and $T$ satisfy condition (3.2) of Theorem 3.1. In this example only mapping $A$ is continuous and so condition (3.3) is satisfied.

Let us consider a decreasing sequence $\left\{x_{n}\right\}$ such that $\lim _{n \rightarrow \infty} x_{n}=3$. Then $\lim _{n \rightarrow \infty}$ $A x_{n}=\lim _{n \rightarrow \infty} S x_{n}=1$ and $\lim _{n \rightarrow \infty}\left\|A S x_{n}-S A x_{n}\right\|=0$. Thus the pair $\{A, S\}$ is compatible. Similarly the pair $\{B, T\}$ is also compatible.

We see that condition (3.1) is satisfied if $\phi$ is similar to that of Example 1 or Example 2 or Example 3. Here we take $k \in[0.5,1)$ in Example 1, $a \in[0.3,1)$ in Example 2 and $c_{1}=0.8, c_{2}=0.1$ in Example 3.

Thus $A, B, S$ and $T$ satisfy all the conditions of Theorem 3.1 and Corollary 3.2 and have a unique common fixed point $x=1$.

For a function $f:(X,\|\cdot\|) \rightarrow(X,\|\cdot\|)$ we denote $F_{f}=\{x \in X: x=f(x)\}$.

Theorem 3.3. Let $A, B, S$ and $T$ be mappings from a normed linear space $(X,\|\cdot\|)$ into itself. If the inequality (3.1) holds for $x, y$ in $X$ then $\left(F_{S} \cap F_{T}\right) \cap F_{A}=\left(F_{S} \cap F_{T}\right) \cap F_{B}$.

Proof. Let $x \in\left(F_{S} \cap F_{T}\right) \cap F_{A}$. Then by (3.1), we have

$$
\begin{aligned}
\phi & (\|A x-B x\|,\|S x-T x\|,\|S x-A x\|,\|T x-B x\|, \\
& (1 / 2)(\|S x-A x\|+\|T x-B x\|),\|T x-A x\|) \leq 0 . \\
= & \phi(\|x-B x\|, 0,0,\|x-B x\|,(1 / 2)\|x-B x\|, 0) \leq 0,
\end{aligned}
$$


which implies, by $\left(\phi_{a}\right)$ that $x=B x$. Thus

$$
\left(F_{S} \cap F_{T}\right) \cap F_{A} \subset\left(F_{S} \cap F_{T}\right) \cap F_{B} .
$$

Similarly, we have by $\left(\phi_{b}\right)$, that

$$
\left(F_{S} \cap F_{T}\right) \cap F_{B} \subset\left(F_{S} \cap F_{T}\right) \cap F_{A} .
$$

Remark. Theorem 3.3 is true if we replace the condition (3.1) by (3.5) or (3.6) or (3.7).

Theorem 3.1 implies following one.

Theorem 3.4. Let $S, T$ and $\left\{A_{i}\right\}_{i \in N}$ be mappings from a Banach space $(X,\|\cdot\|)$ into itself such that

$$
\begin{gathered}
A_{2}(X) \subset S(X) \text { and } A_{1}(X) \subset T(X), \\
\text { one of } S, T, A_{1} \text { and } A_{2} \text { is continuous, } \\
\text { the pairs }\left(A_{1}, S\right) \text { and }\left(A_{2}, T\right) \text { are compatible, }
\end{gathered}
$$

the inequality

$$
\begin{gathered}
\phi\left(\left\|A_{i} x-A_{i+1} y\right\|,\|S x-T y\|,\left\|S x-A_{i} x\right\|,\left(\left\|T y-A_{i+1} y\right\|,\right.\right. \\
(1 / 2)\left(\left(\left\|S x-A_{i} x\right\|+\left\|T y-A_{i+1} y\right\|\right),\left\|A y-A_{i} x\right\|\right) \leq 0
\end{gathered}
$$

holds for each $x, y \in X$, for all $i \in N$ and $\phi \in \Phi$. Then $S, T$ and $\left\{A_{i}\right\}_{i \in N}$ have a unique common fixed point.

\section{References}

[1] G. Jungck, Compatible mappings and common fixed points, Internat. J. Math. Math. Sci. 9(1986), 771-779.

[2] G. Jungck, Compatible mappings and common fixed points (2), Internat. J. Math. and Math. Sci. 11(1988), 285-288.

[3] G. Jungck, Common fixed points for commuting and compatible maps on compacta, Proc. Amer. Math. Soc. 103(1988), 977-983.

[4] S. M. Kang, Y. J. Cho and G. Jungck, Common fixed points of compatible mappings, Internat. J. Math. and Math. Sci. 13(1990), 61-66.

[5] S. M. Kang and J. W. Rye, A commmon fixed point theorem for compatible mappings, Math. Japonica 35(1990), 153-157.

[6] V. Popa, Theorems of unique fixed point for expansion mappings, Demonstratio Math. 23(1990), 213-218.

[7] V. Popa, Common fixed points of compatible mappings, Demons. Math. 26 (3-4) (1993), 802-809.

[8] V. Popa, Some fixed point theorems for compatible mappings satisfying an implicit relation, Demonstratio Math. 32 (1999), 157-163. 
[9] B. E. Rhoades, Some fixed point theorems for pairs of mappings, Jnanabha 15(1985), 151156.

[10] S. Sessa, On weak commutativity condition of mappings in a fixed point consideration, Publ. Inst. Math. 32 (46) (1986), 149-153.

[11] S. Sessa, and B. Fisher, Common fixed points of weakly commuting mappings, Bull. Polish. Acad. Sci. Math. 36 (1987), 345-349.

[12] S. Sessa, B. E. Rhoades and M. S. Khan, On common fixed points of compatible mappings in metric and Banach spaces, Iternat. J. Math. Sci. 11 (2) (1988), 375-392.

[13] Sushil Sharma and P. C. Patidar, On common fixed point theorem of four mappings, Bull. Mal. Math. Soc. (accepted).

[14] S. Z. Wong, B. Y. Li, Z. M. Gao and K. Iseki, Some fixed point theorems on expansion mappings, Math. Japonica 29 (1984), 631-636.

Department of Mathematics, Madhav Science College, Ujjain (M. P.), India.

E-mail:sksharma2005@yahoo.com

Department of Mathematics, Govt. Arts and Science P. G. College, Ratlam (M. P.), India.

E-mail:bhavnadeshpande@yahoo.com 